\title{
Empagliflozin as an adjunctive therapy for type 1 diabetes
}

\author{
Richard J. MacIsaac ${ }^{1,2}$, Melissa H. Lee ${ }^{1,2}$, Sybil A. McAuley ${ }^{1,2}$, Glenn M. Ward ${ }^{1,2}$, David N. O’Neal ${ }^{1,2}$ \\ ${ }^{1}$ Department of Endocrinology \& Diabetes, St Vincent's Hospital, Melbourne, Victoria, Australia; ${ }^{2}$ Department of Medicine, University of \\ Melbourne, Victoria, Australia \\ Correspondence to: Professor Richard J. MacIsaac, Bsc (HONS), PhD, MBBS, FRACP. Director, Department of Endocrinology \& Diabetes, \\ St Vincent's Health, Melbourne, PO Box 2900, Fitzroy, Victoria 3065, Australia. Email: Richard.MacIsaac@svhm.org.au. \\ Comment on: Rosenstock J, Marquard J, Laffel LM, et al. Empagliflozin as Adjunctive to Insulin Therapy in Type 1 Diabetes: The EASE Trials. \\ Diabetes Care 2018;41:2560-9.
}

Submitted Dec 16, 2018. Accepted for publication Dec 18, 2018.

doi: $10.21037 / \mathrm{atm} .2018 .12 .52$

View this article at: http://dx.doi.org/10.21037/atm.2018.12.52

Long-term complications of type 1 diabetes (T1D) due to exposure to chronic hyperglycaemia, including blindness, amputations, cardiovascular disease and kidney failure, remain a fear for many people living with T1D. Intensive insulin therapy reduces the risk of complications, but the majority of people with T1D fail to achieve recommended glycaemic targets (1-3). Furthermore, long-term intensive insulin therapy is associated with weight gain, a relevant side-effect as a significant proportion of people with T1D are now overweight (4).

Acute hyperglycaemia can also be associated with the development of complications such as diabetic ketoacidosis (DKA). In an attempt to reduce glucose levels and the risk of complications, many people with T1D live with the daily struggle of attempting to avoid hypoglycaemia (3). Therefore, maintaining glucose levels within acceptable ranges represents a stressful and time-consuming balancing act that people with T1D face every day.

There have been significant recent advances in insulin delivery and glucose sensing that aim to assist people with T1D addresses the challenge of maintaining glucose levels within recommended targets. These include continuous subcutaneous insulin infusion (pump therapy), continuous glucose monitoring (CGM) and integration of these two components using "closed-loop" or "artificial pancreas" technology. Whilst clinical trials suggest that these technologies may help people to avoid hypoglycaemia and maintain glucose levels within recommended ranges, they are currently not available to the majority of people with T1D and do not negate all the problems associated with exogenous insulin delivery. Whole pancreas and islet cell transplantation are also available in some specialised centres in the world; however, access is currently limited to a small proportion of people with T1D, and transplantation is associated with major disadvantages including the risks associated with the use of immunosuppressive medications (5-7).

The use of adjunct non-insulin therapies has been proposed as a means of helping people with T1D improve their metabolic profile. Ideal adjunctive therapies should have an insulin-independent mechanism of glucose lowering and provide additional benefits such as reduced glycaemic variability with hypoglycaemia reduction, weight loss and other improvements in cardiovascular health. Importantly, the above must be achieved by therapies that have an acceptable safety profile (8).

Currently, pramlintide (an amylin analogue) is the only non-insulin therapy approved for use in T1D, but it is only available in the USA. Due to its need for pre-prandial dosing and the common side-effect of nausea, uptake has been limited. Metformin, glucagon like peptide-1 (GLP-1) receptor agonists and dipeptidyl peptidase-4 (DPP-4) enzyme inhibitors have also been studied in the above context but have failed to show consistent improvements in glycaemia or cardiovascular health (8). A number of recent studies have therefore examined the efficacy and safety of medications that belong to the newest class of glucose lowering therapies available, the sodium-glucose cotransporter inhibitors, in T1D (9).

There are two sodium-glucose cotransporters in the body. SGLT2 mediates high-capacity glucose uptake in the early proximal tubule, and SGLT2 inhibitors, via their ability to promote glycosuria, have been developed as 
glucose lowering medications. As well as having a glucoselowering effect, SGLT2 inhibitors also reduce blood pressure, promote weight loss and reduce uric acid levels. In addition, the SGLT2 inhibitors empagliflozin, canagliflozin and dapagliflozin have been shown to have cardiovascular and renal protective effects in people with type 2 diabetes via mechanisms that have yet to be fully elucidated (10-12). A type 1 SGLT (SGLT1) is also found in the intestine and its inhibition reduces glucose levels mainly through delaying post-prandial hyperglycaemia (13). Outcome studies for SGLT1 or dual SGLT1/2 inhibitors have yet to be performed.

The main disadvantage of the mode of action of SGLT2 inhibitors is that their effectiveness for lowering glucose levels is dependent on renal function; hence, they are not recommended for use in patients with reduced glomerular filtration rate. Potential side-effects or concerns related to the use of SGLT2 inhibitors include increased rates of urinary tract infections, genital tract infections, postural hypotension, DKA, acute kidney injury and possible increased rates of fractures (14). As expected, the main sideeffects with SGLT1 inhibition are gastrointestinal-related and manifest as increased rates of diarrhoea (15).

DKA has become an increasingly recognised side-effect of SGLT2 inhibitor use among people with type 2 diabetes, particularly during times of metabolic stress such as prolonged fasting and/or the presence of inter-current illness (16). Many cases of SGLT2 inhibition-associated DKA also appear to occur with only minor elevations in blood glucose levels (euglycaemic DKA). Indeed, the product information for SGLT2 inhibitors and several alerts issued by various authorities and societies have highlighted the above risks. In retrospect, given one of the postulated mechanisms of action of SGLT inhibition is to promote ketone body formation, the increased risk of DKA with use of medications that inhibit SGLT should not be unexpected (17). Not surprisingly, the risk of DKA has emerged as the main safety concern regarding the use of SGLT inhibitors in T1D.

Recently, the results of the EASE (Empagliflozin as an Adjunctive to inSulin thErapy) trials which investigated the potential benefits and risks associated with the use of empagliflozin in T1D have been published as a combined report in the 2018 December edition of Diabetes Care (18). Of note, the EASE trials contained an arm that involved the use of a very low dose of empagliflozin $(2.5 \mathrm{mg})$, below that used in clinical practice for type 2 diabetes, to specifically examine the question as to whether the risk for DKA could be ameliorated whilst still maintaining some of the expected metabolic improvements of this medication. The results of two trials were reported. The EASE-2 trial involved the use of a daily $10 \mathrm{mg}(\mathrm{n}=243)$ or $25 \mathrm{mg}(\mathrm{n}=244)$ of empagliflozin (doses currently approved for the treatment of type 2 diabetes) compared with placebo $(n=243)$ for 52 weeks, whereas EASE-3 involved the daily use of $2.5 \mathrm{mg}(\mathrm{n}=241)$, $10 \mathrm{mg}$ (248) or $25 \mathrm{mg}(\mathrm{n}=245)$ of empagliflozin compared with placebo $(n=241)$ for 26 weeks. Outcomes of interest included changes in HbA1c levels, rates of hypoglycaemia, CGM time in range $>3.9$ to $\leq 10 \mathrm{mmol} / \mathrm{L}$, weight, blood pressure and rates of DKA.

The mean baseline HbA1c level for patients in the EASE trials was $8.1 \%(65 \mathrm{mmol} / \mathrm{mol})$. Before the randomised portion of the trial commenced, patients underwent an investigator guided insulin intensification period and a placebo run-in period to assess adherence. At the time of randomisation, insulin doses were reduced by up to $10 \%$ if $\mathrm{HbAlc}$ was $<8.0 \%(64 \mathrm{mmol} / \mathrm{mol})$ to reduce the risk of hypoglycaemia. All patients were educated about the risks of DKA and were supplied a blood glucose monitoring device that was also capable of measuring blood ketones [beta-hydroxybutyrate (BHB)]. Patients were also educated on ketone monitoring when feeling unwell, irrespective of their glucose level, and to seek medical care in the case that BHB levels were $>1.5 \mathrm{mmol} / \mathrm{L}$.

The placebo corrected changes in HbAlc from baseline at 52 weeks for EASE-2 were $-0.39 \%$ for $10 \mathrm{mg}$ and $-0.45 \%$ for $25 \mathrm{mg}$ empagliflozin (both $\mathrm{P}<0.0001$ ). Whilst the placebo corrected changes in HbAlc from baseline at 26 weeks for EASE-3 were $-0.28 \%$ for $2.5 \mathrm{mg},-0.45 \%$ for $10 \mathrm{mg}$ and $-0.52 \%$ for $25 \mathrm{mg}$ of empagliflozin (all $\mathrm{P}<0.0001$ ). Empagliflozin 2.5, 10 and $25 \mathrm{mg}$ reduced mean weight by $-1.8,-3.0$ and $-3.4 \mathrm{~kg}$, respectively (all $\mathrm{P}<0.0001$ ), increased glucose time-in-range by $+1.0,+2.9$, +3.1 hours per day, respectively $(\mathrm{P}<0.0001$ for 10 and $25 \mathrm{mg}$ only), lowered total daily insulin dose by $-6.4 \%,-13.3 \%$ and $-12.7 \%$ (all $\mathrm{P}<0.0001)$ and decreased systolic blood pressure by $-2.1,-3.9,-3.7 \mathrm{mmHg}$, respectively $(\mathrm{P}<0.05$ for $2.5 \mathrm{mg}$, $\mathrm{P}<0.0001$ for 10 and $25 \mathrm{mg}$ ). Severe hypoglycaemia was rare and occurred with equal frequency with placebo and all doses of empagliflozin, whilst genital infections were more frequent on empagliflozin $(5.4 \%$ for $2.5 \mathrm{mg}, 12.8 \%$ for $10 \mathrm{mg}$ and $14.3 \%$ for $25 \mathrm{mg}$ ) compared with placebo (2.5\%).

Adjudicated DKA occurred more with empagliflozin $10 \mathrm{mg}(4.3 \%)$ and $25 \mathrm{mg}(3.3 \%)$ but was comparable between empagliflozin $2.5 \mathrm{mg}(0.8 \%)$ and placebo (1.2\%). An exploratory post hoc net clinical benefit analysis found that a greater proportion of patients on empagliflozin 
Table 1 Comparison of HbA1c change and rates of DKA reported in major trials of SGLT inhibition in T1D

\begin{tabular}{|c|c|c|c|c|}
\hline Trial (drug and reference) & Participants (N) & Study duration (weeks) & $\mathrm{HbA} 1 \mathrm{C}$ change vs. placebo (\%) & DKA events (\%) \\
\hline Empagliflozin 10 mg & & & -0.39 & $4.3^{*}$ \\
\hline Empagliflozin 25 mg & & & -0.45 & $3.3^{*}$ \\
\hline Placebo & & & - & 1.2 \\
\hline Empagliflozin $2.5 \mathrm{mg}$ & & & -0.28 & 0.8 \\
\hline Empagliflozin 10 mg & & & -0.45 & $4.3^{*}$ \\
\hline Empagliflozin 25 mg & & & -0.52 & $3.3^{*}$ \\
\hline Placebo & & & - & 1.2 \\
\hline Dapagliflozin 10 mg & & & -0.36 & 3.4 \\
\hline Placebo & & & - & 1.9 \\
\hline DEPICT-2 (18) & 813 & 24 & & \\
\hline Dapagliflozin 5 mg & & & -0.37 & 2.6 \\
\hline Dapagliflozin 10 mg & & & -0.42 & 2.2 \\
\hline Placebo & & & - & 0 \\
\hline inTandem-3 (15) & 1,402 & 24 & & \\
\hline Sotagliflozin 400 mg & & & -0.46 & 3.9 \\
\hline
\end{tabular}

*, participants taking 10 and $25 \mathrm{mg}$ of empagliflozin in the EASE-2 and 3 programs were pooled for this analysis. EASE, empagliflozin as Adjunctive to Insulin Therapy Program. DEPICT, efficacy and Safety of Dapagliflozin in Patients with Inadequately Controlled T1D. The results of a shorter DEPICT-1 trial that ran for 24 weeks (20) have been omitted for clarity. inTandem-3, A Phase 3 Study to Evaluate the Safety of Sotagliflozin in Patients with T1D Who Have Inadequate Glycaemic Control with Insulin Therapy Alone. Other inTandem trials of sotagliflozin have been omitted for clarity. DKA, diabetic ketoacidosis; SGLT, sodium glucose co-transporters.

achieved an end point that included a HbA1c reduction of at least $-0.5 \%$ without weight gain, DKA or severe hypoglycaemia compared with placebo $(+19 \%$ for $2.5 \mathrm{mg}$, $+27 \%$ for $10 \mathrm{mg}$ and $+31 \%$ for $25 \mathrm{mg}$, all $\mathrm{P}<0.001$ ).

As Table 1 shows, the results of the EASE trials are in general agreement with other major trials of SGLT inhibitors in T1D. These trials all had initial HbA1c values ranging between $7.9 \%$ and $8.5 \%$ (63 to $69 \mathrm{mmol} / \mathrm{mol}$ ), study durations of 18 to 52 weeks and involved 351 to 1,402 participants. They including the DEPICT (Efficacy and Safety of Dapagliflozin in Patients with Inadequately Controlled Type 1 diabetes) trials $(19,21)$ and the inTandem-3 (A Phase 3 Study to Evaluate the Safety of Sotagliflozin in Patients With Type 1 Diabetes Who Have
Inadequate Glycaemic Control With Insulin Therapy Alone) trial (15). Dapagliflozin is commercially available and, like empagliflozin, is an SGLT2 inhibitor, whereas sotagliflozin is a co-inhibitor of sodium-glucose transporters type 1 and 2 and is not yet commercially available. A shorter duration trial (18 weeks) of canagliflozin has also reported similar decreases in HbA1c levels and increased rates of DKA (4.3\% for $100 \mathrm{mg}$ and $6.0 \%$ for $300 \mathrm{mg}$ compared with $0 \%$ for placebo) (22).

The results of these trials have provided shortterm evidence that blocking sodium-glucose transport is potentially an attractive adjunct therapy to insulin in T1D. All trials have shown improvements in HbA1c levels and reductions in weight. The DEPICT-2 and EASE-3 
trials also show increased glucose time in range $(18,19)$. Furthermore, rates of hypoglycaemia were shown to be lower in SGLT2 inhibitor-treated participants. However, these benefits were negated by increased rates of DKA. The only exception to this was seen with the use of very low dose of empagliflozin $(2.5 \mathrm{mg})$ in EASE-3. At this dose, rates of DKA were similar to placebo-treated participants, but more participants treated with $2.5 \mathrm{mg}$ empagliflozin per day achieved a combined endpoint of HA1c reduction $>0.5 \%$ without weight gain, severe hypoglycaemia or DKA than placebo-treated participants $(23.6 \%$ versus $4.2 \%$, $\mathrm{P}<0.0001)$. Despite this, it is still worth noting that the metabolic benefits of this low dose therapy were smaller than those with the 10 or $25 \mathrm{mg}$ doses of empagliflozin (18).

Currently, SGLT2 inhibition is not an approved treatment for people with T1D. However, the "offlabel" use of SGLT2 inhibition for T1D is becoming more common in clinical practice. In recognition of this, protocols and clinical recommendations regarding approaches to reducing the risk of DKA have recently been published. Common themes that are highlighted to avoid the development of DKA include adequate patient education regarding risk of DKA, in particular, highlighting that it can occur in the setting of normal or minimallyraised glucose levels. Hence there is a need to rely directly on blood ketone levels rather than glucose levels to assess for the possible development of ketosis. Of note, urinary ketone measurement is not recommended to gauge the degree of ketosis in the setting of SGLT inhibitor use (23). In addition, patients should be aware of the need to cease SGLT inhibitor therapy during intercurrent illness or when fasting for surgery.

One set of recommendations has highlighted the importance of correct patient selection for the initiation of SGLT inhibitor therapy and suggests that these medications should be avoided in patients with T1D who have HbA1c levels $>9 \%(75 \mathrm{mmol} / \mathrm{mol})$ and a history of DKA (9). When SGLT2 inhibitors are used in T1D, approaches to minimise DKA risk include using the lowest dose of SGLT2 inhibitor therapy required to achieve clinical benefit, reassessment of insulin-to-carbohydrate ratios, the ability and means to measure blood ketone levels and advice to test blood ketone levels if sick (irrespective of blood glucose levels), as mentioned above (9). Also, patients with T1D taking SGLT inhibitors should avoid excess alcohol and very low carbohydrate or ketogenic diets.

Furthermore, it has been suggested that patients on insulin pump therapy who may possibly be at increased risk for DKA should be very carefully selected for the initiation of SGLT2 inhibitor therapy. Insulin pump-treated patients are theoretically at an increased risk for the development of DKA compared with multi-dose injection patients. As rapid acting insulin alone is used in pump therapy, the absence of longacting basal insulin can be detrimental when insulin delivery is interrupted in those on pump therapy, leading to rapid rises in blood glucose levels and the development of ketosis.

However, one very small study of otherwise well patients with T1D on pump therapy suggested that after starting an SGLT2 inhibitor and after short-term pump suspension, rates of ketosis are not accelerated and blood glucose levels rise at a slower rate compared with pump suspension studies performed prior to starting an SGLT2 inhibitor (25). This study also emphasises the fact that failure of patients with T1D on SGLT2 inhibitors to promptly recognise early metabolic disturbances in the setting of a very gradual rise in blood glucose levels may be one of the key reasons why DKA develops.

Once ketosis has developed, the STICH (Stop, Inject, Carbohydrate and Hydrate) protocol has been developed to mitigate the risk of progressing to DKA for people with T1D on adjunctive treatment with SGLT inhibitors (23). The key steps involved in this protocol include (I) verifying ketosis by identification of early symptoms and appropriate testing and identifying conditions that cause ketosis, (II) stopping the SGLT inhibitor, injecting bolus insulin, consuming $30 \mathrm{~g}$ of carbohydrate and hydrating, (III) rechecking ketone levels every 3-4 hours and (IV) seeking medical care if ketosis does not resolve or if symptoms of DKA appear such as abdominal pain, nausea and vomiting.

The protocol acknowledges that there may be a considerable delay between ceasing an SGLT inhibitor and the development of ketosis. Indeed, some guidelines are recommending cessation of an SGLT2 inhibitor 3 days prior to surgery because of this risk (25). The STICH protocol also suggests that patients carry a wallet card that contains information on how SGLT inhibitor associated DKA may differ from standard DKA. Guidance on how to correct SGLT-associated DKA, that is, the administration of rapid-acting insulin regardless of blood glucose levels and the administration of carbohydrates is also emphasised in the information contained on the wallet card.

In summary, SGLT inhibition is emerging as a very promising adjunctive therapy in T1D, but the risk of DKA remains a significant barrier to the widespread uptake of this class of medication. Further long-term trials examining the thresholds for low-dose SGLT inhibition that can achieve 
clinically meaningful outcomes with an acceptable risk of DKA appear warranted. The risk of DKA may also need to be considered in the context of protection from renal and cardiovascular complications if these proven benefits of SGLT2 inhibition in type 2 diabetes are demonstrated in T1D. In the interim, patient selection and education about the risks of DKA are key to preventing the development of ketosis when SGLT inhibitors are used "off label" in T1D.

\section{Acknowledgements}

None.

\section{Footnote}

Conflicts of Interest: Professor Richard MacIsaac has received honoraria and travel support for lectures from Eli lily, Novo Nordisk, Sanofi Aventis, Astra Zeneca, Merck Sharp \& Dohme and Norvartis. He has received research grants from Novo Nordisk and Sevier. Dr. Sybil A. McAuley has received speaker honoraria for lectures from Novo Nordisk and Eli Lilly; travel support from Novo Nordisk; and research support from Medtronic. David O'Neal: has received travel support from Novo-Nordisk; honoraria for lectures from Novo Nordisk, Sanofi Aventis, Merck Sharp \& Dohme, Medtronic and Dexcom; He has received research grants and research support from Novo Nordisk, Medtronic, Roche, Sanofi-Aventis; Abbott, and Eli-Lily. He has been on advisory boards for Novo-Nordisk, Medtronic, Abbott and Sanofi-Aventis.

\section{References}

1. Nathan DM, Bayless M, Cleary P, et al. Diabetes control and complications trial/epidemiology of diabetes interventions and complications study at 30 years: advances and contributions. Diabetes 2013;62:3976-86.

2. McKnight JA, Wild SH, Lamb MJ, et al. Glycaemic control of Type 1 diabetes in clinical practice early in the 21st century: an international comparison. Diabet Med 2015;32:1036-50.

3. Miller KM, Foster NC, Beck RW, et al. Current state of type 1 diabetes treatment in the U.S.: updated data from the T1D Exchange clinic registry. Diabetes Care 2015;38:971-8.

4. Conway B, Miller RG, Costacou T, et al. Temporal patterns in overweight and obesity in Type 1 diabetes. Diabet Med 2010;27:398-404.
5. Pickup JC. Is insulin pump therapy effective in Type 1 diabetes? Diabet Med. 2018. [Epub ahead of print].

6. Wood A, O'Neal D, Furler J, et al. Continuous glucose monitoring: a review of the evidence, opportunities for future use and ongoing challenges. Intern Med J 2018;48:499-508.

7. Nijhoff MF, de Koning EJP. Artificial Pancreas or Novel Beta-Cell Replacement Therapies: a Race for Optimal Glycemic Control? Curr Diab Rep 2018;18:110.

8. Frandsen CS, Dejgaard TF, Madsbad S. Non-insulin drugs to treat hyperglycaemia in type 1 diabetes mellitus. Lancet Diabetes Endocrinol 2016;4:766-80.

9. McCrimmon RJ, Henry RR. SGLT inhibitor adjunct therapy in type 1 diabetes. Diabetologia 2018;61:2126-33.

10. Heerspink HJ, Perkins BA, Fitchett DH, et al. Sodium Glucose Cotransporter 2 Inhibitors in the Treatment of Diabetes Mellitus: Cardiovascular and Kidney Effects, Potential Mechanisms, and Clinical Applications. Circulation 2016;134:752-72.

11. Verma S, McMurray JJV. SGLT2 inhibitors and mechanisms of cardiovascular benefit: a state-of-the-art review. Diabetologia 2018;61:2108-17.

12. Wiviott SD, Raz I, Bonaca MP, et al. Dapagliflozin and Cardiovascular Outcomes in Type 2 Diabetes. N Engl J Med 2018. [Epub ahead of print].

13. Rieg T, Vallon V. Development of SGLT1 and SGLT2 inhibitors. Diabetologia 2018;61:2079-86.

14. Lupsa BC, Inzucchi SE. Use of SGLT2 inhibitors in type 2 diabetes: weighing the risks and benefits. Diabetologia 2018;61:2118-25.

15. Garg SK, Henry RR, Banks P, et al. Effects of Sotagliflozin Added to Insulin in Patients with Type 1 Diabetes. N Engl J Med 2017;377:2337-48.

16. Meyer EJ, Gabb G, Jesudason D. SGLT2 InhibitorAssociated Euglycemic Diabetic Ketoacidosis: A South Australian Clinical Case Series and Australian Spontaneous Adverse Event Notifications. Diabetes Care 2018;41:e47-9.

17. Ferrannini E, Baldi S, Frascerra S, et al. Shift to Fatty Substrate Utilization in Response to Sodium-Glucose Cotransporter 2 Inhibition in Subjects Without Diabetes and Patients With Type 2 Diabetes. Diabetes 2016;65:1190-5.

18. Rosenstock J, Marquard J, Laffel LM, et al. Empagliflozin as Adjunctive to Insulin Therapy in Type 1 Diabetes: The EASE Trials. Diabetes Care 2018;41:2560-9.

19. Dandona P, Mathieu C, Phillip M, et al. Efficacy and Safety of Dapagliflozin in Patients With Inadequately Controlled Type 1 Diabetes: The DEPICT-1 52-Week 
Study. Diabetes Care 2018;41:2552-9.

20. Dandona P, Mathieu C, Phillip M, et al. Efficacy and safety of dapagliflozin in patients with inadequately controlled type 1 diabetes (DEPICT-1): 24 week results from a multicentre, double-blind, phase 3, randomised controlled trial. Lancet Diabetes Endocrinol 2017;5:864-76.

21. Mathieu C, Dandona P, Gillard P, et al. Efficacy and Safety of Dapagliflozin in Patients With Inadequately Controlled Type 1 Diabetes (the DEPICT-2 Study): 24-Week Results From a Randomized Controlled Trial. Diabetes Care 2018;41:1938-46.

22. Henry RR, Thakkar P, Tong C, et al. Efficacy and Safety of Canagliflozin, a Sodium-Glucose Cotransporter 2 Inhibitor, as Add-on to Insulin in Patients With Type 1 Diabetes. Diabetes Care 2015;38:2258-65.

Cite this article as: MacIsaac RJ, Lee MH, McAuley SA, Ward GM, O’Neal DN. Empagliflozin as an adjunctive therapy for type 1 diabetes. Ann Transl Med 2018;6(Suppl 2):S134. doi: 10.21037/atm.2018.12.52
23. Garg SK, Peters AL, Buse JB, et al. Strategy for Mitigating DKA Risk in Patients with Type 1 Diabetes on Adjunctive Treatment with SGLT Inhibitors: A STICH Protocol. Diabetes Technol Ther 2018;20:571-5.

24. Patel NS, Van Name MA, Cengiz E, et al. Altered Patterns of Early Metabolic Decompensation in Type 1 Diabetes During Treatment with a SGLT2 Inhibitor: An Insulin Pump Suspension Study. Diabetes Technol Ther 2017;19:618-22.

25. Australian Diabetes Society Alert: Severe euglycaemic ketoacidosis with SGLT2 inhibitor use in perioperative period. 2018. Available online: https:// diabetessocietycomau/documents/2018_ALERTADS_SGLT2i_PerioperativeKetoacidosis_v3 final2018_02_14pdf 